\title{
Effect of Epidermal Growth Factor with Silastic Scaffold on Tympanic Membrane Perforations of Chronic Otitis Media
}

\author{
Vikram Kemmannu Bhat ${ }^{1,}$ Suhas Suresh Desai ${ }^{1,}$ Darshan Hiremath ${ }^{1}$, \\ ${ }^{1}$ Department of Otolaryngology, Head and Neck Surgery, Karnataka \\ Institute of Medical Sciences, Hubli, Karnataka, India \\ Address for correspondence Vikram Kemmannu Bhat, MS, DNB, \\ MNAMS, PhD, Department of Otolaryngology, Head and Neck \\ surgery, Karnataka Institute of Medical Sciences, Vidyanagar, \\ Hubli-580021, Karnataka, India (e-mail: vikram.ent@gmail.com).
}

\begin{abstract}
Aims The burden of chronic otitis media continues to be high, especially in developing countries. The demand for corrective procedure of surgical myringoplasty, which is considered the gold standard for tympanic membrane perforations, is also growing. Hence, there is a need to innovate simpler alternative office procedures that could save effort, time, waiting period, and expenditure.

Materials and Methods This prospective study of 84 patients with mucosal chronic otitis media was undertaken in a tertiary referral hospital. All patients underwent eustachian tube function test, diagnostic nasal endoscopy, and pure tone audiometry. The size of the perforation was assessed endoscopically. All perforations were freshened first with silver nitrate solution. Silastic pieces were placed medial and lateral to the perforation with epidermal growth factor gel in between. Patients were followed up for 3 months.

Results The perforations were classified into three groups (healed, partially healed, and nonhealed) based on the treatment response at the end of the first and third

Keywords

- otitis media

- silastic

- epidermal growth factor

- myringoplasty follow-up months. Completely healed and partially healed perforations were considered as "success" and were found to be $79 \%$ in this study. Significant association was found between healing status and type of anesthesia. However, the disease stage, size of perforation, and the duration of procedure did not affect the healing status.

Conclusion Silastic enhanced myringoplasty with epidermal growth factor is a simple, effective, and economical procedure that can become an alternative to surgical myringoplasty for chronic otitis media.
\end{abstract}

\section{Introduction}

The surgical closure of tympanic membrane perforation of chronic otitis media (COM) is an elaborate process as it is a time-consuming reconstructive procedure needing the harvesting of a graft. Given the high load of COM cases, especially in the developing countries, simpler procedures are required to tackle this condition, as most of them would not heal and close spontaneously. Left untreated, there would instead be a further deterioration of the condition in terms of
Published online September 14, 2021
DOI https://doi.org/ $10.1055 / \mathrm{s}-0041-1735386$ ISSN 2581-9607

\footnotetext{
(C) 2021. Indian Society of Otology.

This is an open access article published by Thieme under the terms of the Creative Commons Attribution-NonDerivative-NonCommercial-License, permitting copying and reproduction so long as the original work is given appropriate credit. Contents may not be used for commercial purposes, or adapted, remixed, transformed or built upon. (https://creativecommons.org/licenses/by-nc-nd/4.0/). Thieme Medical and Scientific Publishers Pvt. Ltd. A-12, 2nd Floor, Sector 2, Noida-201301 UP, India
} 
destruction of hearing components and subsequent worsening of hearing loss. Hence, the need for a simple office procedure that could close or at least reduce most perforations of COM. This article is the third in our series of experiments on the effect of epidermal growth factor (EGF) on the healing of tympanic membrane perforations in humans. ${ }^{1,2}$

\section{Materials and Methods}

This pilot study was undertaken in a tertiary referral public hospital in a span of 1 year, after obtaining the necessary clearances from the institutional ethics review board. Every consecutive eligible patient (age 15-70 years) was recruited into the study (prospective case series). The inclusion criteria included small, medium, and large perforations of the pars tensa due to mucosal COM, trauma, or previous myringoplasty failures. However, patients with pinhole, total or subtotal perforations, actively discharging COM, squamosal COM, ossicular chain discontinuity, Eustachian tube malfunction, craniofacial anomalies, and the mentally disabled were excluded. Written informed consent for the procedure was taken from all. They underwent diagnostic nasal endoscopy, Eustachian tube function tests using Eustachian barotubometer, ${ }^{3,4}$ otoendoscopy, and pure tone audiometry (PTA) before the procedure. Pure tone averages were determined for air conductions at 500, 1,000, 2,000, and 4,000 Hz.

The size of the perforation was assessed endoscopically using a right-angled graduated $(\mathrm{mm})$ thin hook. Two perpendicular diameters of perforation were measured and noted three times during the study (before the procedure, first, and third postprocedural months).

\section{Technical Methods}

Injection glycopyrrolate $2 \mathrm{mg}$ was given intramuscularly half an hour before the procedure. Most of the patients underwent the procedure under topical anesthesia (TA) wherein two puffs of lignocaine $10 \%$ solution were sprayed into the ear canal. However, a few of them who refused TA had to undergo the procedure under monitored anesthesia care (MAC). Under the microscopic or endoscopic vision, the perforation margins were cauterized using an ear probe dipped in silver nitrate solution ( $2 \mathrm{~g}$ of silver nitrate in $2 \mathrm{~mL}$ of sterile distilled water). Excess silver nitrate left behind was neutralized with a normal saline ear wash followed by suctioning. Then, two or three pieces of dry gel foam (absorbable gelatin sponge, Virchow Biotech Private Ltd; Rangareddy, Telangana, India) were placed inside the middle ear through the perforation. A thin $(0.005 \mathrm{~mm})$ circular piece (diameter $4 \mathrm{~mm}$ ) of silastic (silicone elastomer: polydimethylsiloxane, Dow Corning Ltd, Midland, Michigan, United States) was placed under the margins of the perforation over the gel foam. One gram of EGF gel (Regen-D $150 \mu \mathrm{g} / \mathrm{g}$ manufactured by Bharat Biotech International Limited, Hyderabad, Telangana, India) was applied on the margins of the perforation and the inner silastic piece. Then, a slightly larger silastic piece $(0.01 \mathrm{~mm}$ thick, diameter $6 \mathrm{~mm}$ ) was placed above the gel and the pars tensa to cover and conceal the perforation completely. The external ear canal (EAC) was packed with a small piece of povidone-iodine tulle (Povi 10 containing povidone-iodine IP $10 \% \mathrm{w} / \mathrm{w}$, ornidazole IP $1 \% \mathrm{w} / \mathrm{w}$ manufactured by Nemus Pharmaceuticals Pvt Ltd, India). Paraffin-smeared cotton ball was placed over the tulle and secured externally with a small piece of sticking plaster. The patient was observed for 1 hour afterward and then sent home. Tablets azithromycin $500 \mathrm{mg}$ daily for 3 days and chlorpheniramine maleate $2 \mathrm{mg}$ thrice daily for 10 days were prescribed. The patient was asked not to sneeze, cough, or blow the cheeks to prevent the migration of silastic pieces. The EAC pack was removed after 15 days. However, the quality of healing and improvement in hearing (PTA) were assessed at 30 and 90 days.

The outcomes assessed were the healing of the perforation (complete, partial, and nonhealed), time taken for procedure, and the type of anesthesia (TA or MAC) required. STATA version 14.0 was used to analyze the results. The level of significance was set at $95 \%$ confidence interval. The observations were analyzed using chi-square test, analysis of variance (ANOVA), and Fisher's exact test.

\section{Observations and Results}

There were 84 patients (males 32, females 52) altogether and the youngest was 12 years old and the oldest 73 years old. The mean age of the patients in the study was $33.35 \pm$ 13.65 years. The patients were classified into three groups (healed, partially healed, and nonhealed) based on the healing of perforation at the end of first and third postoperative months. The outcome was considered a "success" if the perforation closed completely (healed) or reduced in size (partially healed) and a "failure" if the perforation did not change or worsened after the procedure.

In our study of 84 patients, $23(27.4 \%)$ patients had a completely healed tympanic membrane, while 43 (51.2\%) patients had a partially healed tympanic membrane. In 18 (21.4\%) patients tympanic membrane did not heal at all. Hence, the overall successes stood at $79 \%$.

As per - Table 1, the disease status had no impact on the healing status. The perforation size too did not affect the healing status as seen in - Fig. 1 ( $p=0.642$, chi-square test).

In this study, there was a gross disparity in the case numbers in the two groups of anesthesia. This was because the preferred method was TA and MAC was administered only to those who refused the former. There was a significant association between healing status and the type of anesthesia ( - Table 2). As per - Fig. 2, the duration of procedure had no impact over the healing (one-way ANOVA test, $p=0.884$ ), even though the duration was higher with MAC. Time taken for TA procedure was less than MAC which was found to be significant (-Table 3). Two-thirds of the perforations, irrespective of the size, healed within the first month after the procedure. The remaining took longer, with a just significant difference between the groups (-Table 4).

There was no significant association between the size of perforation and the healing status in the first month 
Table 1 Analysis of the disease stage of the middle ear and the healing status

\begin{tabular}{|l|l|l|l|l|}
\hline $\begin{array}{l}\text { Chronic otitis media } \\
(\text { COM) disease stage }\end{array}$ & Healed & Partially healed & Nonhealed & $p$-Value \\
\hline $\begin{array}{l}\text { Inactive } \\
(n=59)\end{array}$ & $15(65.2)$ & $33(76.7)$ & $11(6.1)$ & 0.393 \\
\hline $\begin{array}{l}\text { Quiescent } \\
(n=25)\end{array}$ & $8(34.8)$ & $10(23.3)$ & $7(38.9)$ & \\
\hline
\end{tabular}

${ }^{a}$ Chi-square test.

Table 2 Analysis of the type of anesthesia administered and the healing status

\begin{tabular}{|l|l|l|l|l|}
\hline Anesthesia & Healed & Partially healed & Nonhealed & $p_{\text {-Value }}$ \\
\hline $\begin{array}{l}\text { MAC } \\
(n=9)\end{array}$ & $3(13.0)$ & $1(23)$ & $5(27.8)$ & 0.012 \\
\hline $\begin{array}{l}\text { TA } \\
(n=75)\end{array}$ & $20(86.9)$ & $42(97.7)$ & $13(72.2)$ & \\
\hline
\end{tabular}

Abbreviations: MAC, monitored anesthesia care; TA, topical anesthesia. ${ }^{a}$ Chi-square test.

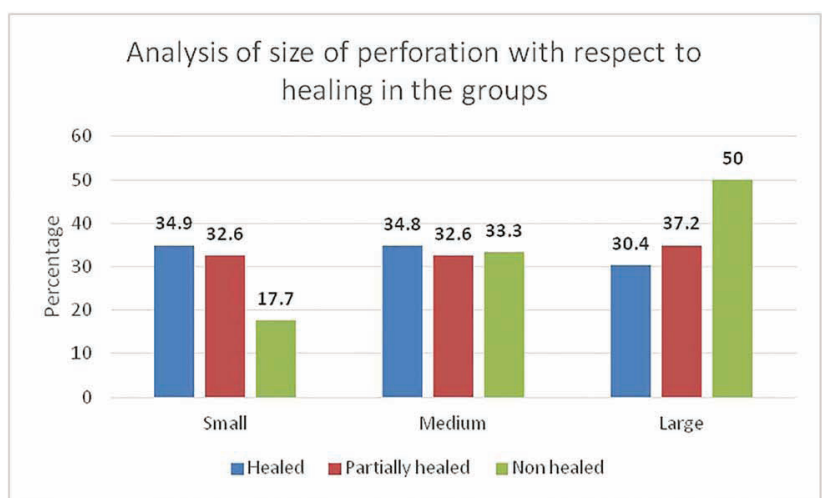

Fig. 1 Bar chart showing the distribution of the size of perforation and its effects on healing.

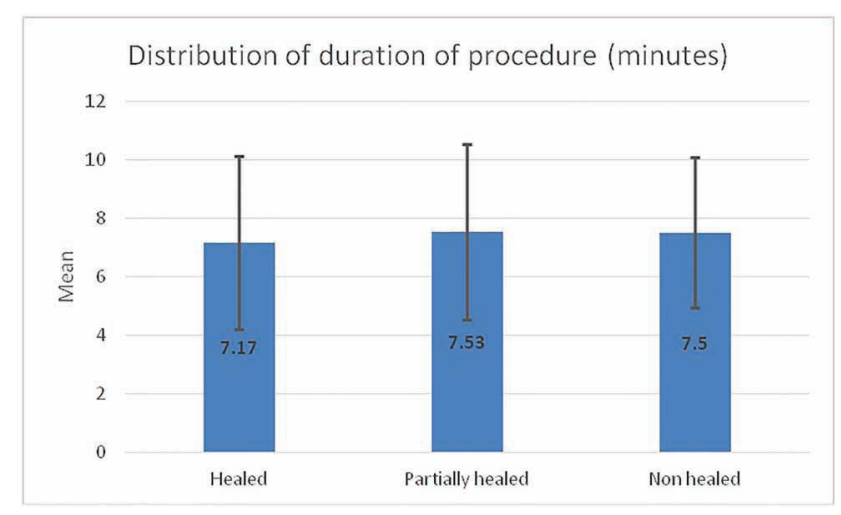

Fig. 2 Bar chart showing the average time taken for the procedure and its impact on healing.

Table 3 Analysis of the duration of the procedure and the type of anesthesia

\begin{tabular}{|c|c|c|c|c|}
\hline Duration of procedure & Total & MAC & TA & $p$-Value ${ }^{a}$ \\
\hline Less than 5 min & 45 & $1(2.2)$ & $44(97.8)$ & \multirow[t]{2}{*}{0.039} \\
\hline 5 to $10 \mathrm{~min}$ & 39 & $6(15.4)$ & $33(84.6)$ & \\
\hline Total & 84 & $7(8.3)$ & $77(91.7)$ & \\
\hline
\end{tabular}

Abbreviations: MAC, monitored anesthesia care; TA, topical anesthesia.

aFisher's exact test.

Table 4 Analysis of the follow-up of the completely healed cases

\begin{tabular}{|l|l|l|l|l|l|}
\hline Follow-up & Total & Small perforation & $\begin{array}{l}\text { Medium } \\
\text { perforation }\end{array}$ & Large perforation & $p_{\text {-Value }}$ \\
\cline { 1 - 5 } First month & 16 & $5(31.3)$ & $6(37.5)$ & $5(31.3)$ & 0.059 \\
\hline Third month & 7 & $2(28.6)$ & $3(42.9)$ & $2(28.6)$ & \\
\hline Total & 23 & $7(30.4)$ & $9(39.1)$ & $7(30.4)$ & \\
\hline
\end{tabular}

${ }^{a}$ Chi-square test.

follow-up (-Table 5). The number of cases that fully healed increased in the last follow-up. Again, there was no significant association between the size of the perforation and healing status in the third month follow-up (-Table 6 ).

\section{Discussion}

COM mucosal type is one of the most common ear diseases presenting in the outpatient clinics of most developing countries. Most of these perforations are not amenable to medical 
Table 5 Analysis of the healing outcomes of the cases at the first month follow-up

\begin{tabular}{|l|l|l|l|l|l|}
\hline $\begin{array}{l}\text { Tympanic } \\
\text { perforations }\end{array}$ & Total & Small & Medium & Large & $p_{\text {-Value }}$ \\
\hline Completely healed & 16 & $5(31.3)$ & $6(37.5)$ & $5(31.3)$ & 0.329 \\
\hline Partially healed & 50 & $17(34)$ & $16(32)$ & $17(34)$ & \\
\hline Nonhealed & 18 & $2(11.1)$ & $6(33.3)$ & $10(55.5)$ & \\
\hline Total & 84 & $24(28.6)$ & $28(33.3)$ & $32(38.1)$ & \\
\hline
\end{tabular}

${ }^{\mathrm{a} C h i-s q u a r e ~ t e s t . ~}$

Table 6 Analysis of the healing outcomes of the cases at the third month follow-up

\begin{tabular}{|l|l|l|l|l|l|}
\hline $\begin{array}{l}\text { Tympanic } \\
\text { perforations }\end{array}$ & Total & Small & Medium & Large & $p_{\text {-Value }}$ \\
\hline Completely healed & 23 & $8(34.8)$ & $8(34.9)$ & $7(30.4)$ & 0.681 \\
\hline Partially healed & 43 & $13(30.2)$ & $14(32.6)$ & $16(37.2)$ & \\
\hline Nonhealed & 18 & $3(16.7)$ & $6(33.3)$ & $9(50.0)$ & \\
\hline Total & 84 & $24(28.6)$ & $28(33.3)$ & $32(39.1)$ & \\
\hline
\end{tabular}

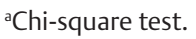

treatment alone. However, many patients do not want a surgical scar in the mastoid region to harvest temporalis fascia. They are also unwilling to get admitted to the hospital and do not want general or infiltrative anesthesia. This attitude of patients is probably because of the painless nature of the tympanic perforation. When this is the case, naturally, patients would consent for a 5-minute office procedure under TA.

The healing effect of EGF has already been studied before and compared against silver nitrate. ${ }^{1}$ It has also been proved to enhance and hasten the closure of perforations during myringoplasty. ${ }^{2}$ EGF is a readymade product that can be used commonly for all patients, unlike the platelet-rich plasma (PRP) that needs to be prepared individually in a centrifuge. ${ }^{5}$ Another advantage of EGF is that it is readily available for repeat procedures if in case needed. However, EGF is heat-labile and needs to be transported only in a cold chain but has a longer shelf life than PRP.

To the best of our knowledge, no such study has been reported in literature and the role of silastic in enhancing the combined effect of EGF and silver nitrate is not studied until now.
In one of our previous studies, the success rate of conventional myringoplasty with and without EGF was 93.33 and 77\%, respectively. ${ }^{2}$ It is evident from - Fig. 1 that the size of perforation had no effect on its healing when both EGF and silastic were used. This outcome might have been due to the silastic sheet medial and lateral to the tympanic membrane perforation. Hence, in the present study, the use of silastic pieces has made possible the healing of larger perforations (72\%) and made them comparable with the smaller ones (87.5\%) even though only $28.57 \%$ of the perforations were small.

The COM stage (quiescent or inactive) did not seem to make a difference for the healing process (-Table $\mathbf{1}$ ). This could be possible because the patients were recruited into the study only after ruling out symptomatic disease in the nose and then subjecting them to Eustachian tube function tests. The overall reduction in the size of perforation after the procedure was most effective when EGF was combined with silastic placement (-Table 7 ). The safety and efficacy of silastic sheeting in the middle ear has already been proved. ${ }^{6}$ Further, silastic sheet myringoplasty has also been tried and a success rate of $54.16 \%$ was found. ${ }^{7}$ Silastic sheet

Table 7 Comparison of the outcomes of the present study with the previous ones

\begin{tabular}{|c|c|c|c|c|}
\hline Healing status & $\begin{array}{l}\text { With EGF, silver nitrate, } \\
\text { and silastic } \\
\text { ( } n=84) \\
\text { (Present study) }\end{array}$ & $\begin{array}{l}\text { With EGF, silver } \\
\text { nitrate }^{-a} \\
(n=42) \\
\text { Group A }\end{array}$ & $\begin{array}{l}\text { With silver nitrate } \\
\text { only }^{-\mathrm{a}} \\
(n=38) \\
\text { Group B }\end{array}$ & $\begin{array}{l}\text { Conventional } \\
\text { myringoplasty } \\
(n=30) \\
\text { Group B }\end{array}$ \\
\hline Completely healed (A) & $23(27.4 \%)$ & $26(86.6 \%)$ & $15(83.3 \%)$ & $8(27 \%)$ \\
\hline $\begin{array}{l}\text { Partially healed } \\
\text { (B) }\end{array}$ & $43(51.2 \%)$ & $4(13.3 \%)$ & $3(16.6 \%)$ & $17(57 \%)$ \\
\hline $\begin{array}{l}\text { Total improved } \\
(A+B)\end{array}$ & $66(79 \%)$ & $30(71.42 \%)$ & $18(47.36 \%)$ & $23(77 \%)$ \\
\hline
\end{tabular}

Abbreviation: EGF, epidermal growth factor.

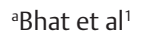

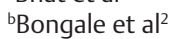


has also been tried to close perforations after removal of Goode-type tympanostomy tubes and found to increase the rate of healing. ${ }^{8}$ Gelfoam has already been used in place of a fat plug to close perforations after freshening their margins. ${ }^{9}$ Cases that did not respond or partially responded after 3 months to this procedure were offered repeat applications with the same settings. We have observed that these repeat procedures too have benefited many patients who otherwise would have had to undergo a surgical myringoplasty under general anesthesia.

As shown in - Table 7, the overall success rate of the present study is higher than previous study ${ }^{1}$ undertaken by us without the use of silastic. However, in the previous study $^{1}$ only small perforations were considered. Comparing the current study with our own previous study, ${ }^{2}$ the overall success rate is comparable with conventional myringoplasty. Hence, the current procedure could be an excellent alternative to many patients who are otherwise unfit for an hour's myringoplasty under general anesthesia like the immunocompromised, cardiac patients, transplant patients, and those with multiple systemic comorbidities. This procedure is also useful for the failures of surgical myringoplasty who do not want to undergo a revision. Besides there being no scar behind the ear, the pain due to temporalis fascia harvest is also absent. ${ }^{10}$ Another great advantage of this procedure is its easy repeatability in nonhealed cases, as it is an office procedure. This procedure is also suitable for large patient gatherings like camps conducted for COM or at the primary health care levels of developing countries.

Lou and colleagues, in a series of cases, claimed $100 \%$ closure of tympanic membrane perforations in 6 months. ${ }^{11,12}$ However, EGF was used as ear drops and applied daily by the patient for 6 months or till the perforation closed. Djamin et al studied the use of EGF in perforations along with amniotic membrane scaffold. ${ }^{13}$ They concluded that the group with EGF had a faster decrease in perforation diameter. Lou et al in a series of two randomized studies, noted the reduced closure time and higher closure rates in the groups receiving EGF for traumatic perforations. ${ }^{14,15}$ However, again EGF drops were used on a daily basis without any scaffolding material. Ramsay et al in 17 patients, found that EGF was safe for use and needed higher concentrations for the closure of perforations. ${ }^{16}$ Jian-Yang et al too found a shorter closure time of traumatic perforations with EGF application in a retrospective review. ${ }^{17}$

\section{Conclusion}

Silastic enhanced myringoplasty with EGF and silver nitrate is an easy, simple, effective, and economical procedure and can become an alternative to surgical myringoplasty for COM. Besides saving time and effort, its easy repeatability makes it more acceptable to the patients, especially the recurrent and the revision cases. The procedure could be particularly beneficial to the developing countries with a high volume of mucosal COM cases waiting for myringoplasty. The size of the perforation and the stage of COM disease had no impact on the healing process of the perforation in this study.

\section{Authors' Contributions}

V.K.B. contributed towards the study idea and design, conceptualization, data collection, manuscript writing, and editing. S.S.D. contributed towards the data collection, data analysis, manuscript writing, and critical review. D.H. contributed towards the data analysis, manuscript writing, editing, and critical review. All authors read and approved the final manuscript.

\section{Conflict of Interest}

All authors declare that there were no conflicts of interest whatsoever in the conduct or writing of this research.

\section{Ethics Approval}

Institutional Ethical Board clearance was obtained for the study

\section{Informed Consent}

This was obtained from all participants both for conducting the study as well as publishing it.

\section{Registration of Study}

This study was registered with the National Clinical Trials Registry [CTRI/2020/04/033027].

\section{References}

1 Bhat VK, Veetil BV, Mathad VK. Effect of silver nitrate and epidermal growth factor on nonhealing tympanic membrane perforations: a randomized controlled study. Ann Otol Neurotol 2018;1:29-33

2 Bongale KR, Bhat VK, Yadav M. Effect of epidermal growth factor on the outcomes of tympanoplasty. Ann Otol Neurotol 2020;3:23-26

3 Bhat VK, Kumar PR, Nag M, Hegde J. Comparison of a Eustachian barotubometer with a tympanometer to evaluate Eustachian tube function in chronic suppurative otitis media. J Otolaryngol Head Neck Surg 2009;38(4):456-461

4 Bhat VK, Marimuthu GS, Herur K, Yadav M. Evaluation of Eustachian tube function in mucosal chronic otitis media: a cost-effective method for developing countries. Ann Otol Neurotol 2018;1:111-114

5 El-Anwar MW, Elnashar I, Foad YA. Platelet-rich plasma myringoplasty: a new office procedure for the repair of small tympanic membrane perforations. Ear Nose Throat J 2017;96(8):312-326

$6 \mathrm{Ng} \mathrm{M}$, Linthicum FH Jr. Long-term effects of Silastic sheeting in the middle ear. Laryngoscope 1992;102(10):1097-1102

7 Chen SL, Yang SW. Factors affecting the treatment outcomes of myringoplasty in patients with small tympanic membrane perforations. Eur Arch Otorhinolaryngol 2019;276(11):3005-3012

8 Courteney-Harris RG, Ford GR, Ganiwalla TM, Mangat KS. Closure of tympanic membrane perforation after the removal of Goode-type tympanostomy tubes: the use of silastic sheeting. J Laryngol Otol 1992;106(11):960-962

9 Niklasson A, Tano K. The Gelfoam ${ }^{\circledR}$ plug: an alternative treatment for small eardrum perforations. Laryngoscope 2011;121(4):782-784

10 Bhat VK, Ajur S, Bongale KR, Sachidananda R. Prevention of craniofacial pain secondary to harvesting of temporalis fascia - a novel technique. J Int Adv Otol 2019;15(3):405-408

11 Lou Z. The effect of epidermal growth factor on the pseudo-healing of traumatic tympanic membrane perforations. Rev Bras Otorrinolaringol (Engl Ed 2021;87(1):53-58

12 Lou ZC, Wei H, Lou ZH. Pretreatment factors affecting traumatic tympanic membrane regeneration therapy using epidermal growth factor. Am J Otolaryngol 2018;39(6):711-718 
13 Djamin R, Aziza R, Rahardjo SP, et al. Topical application of epidermal growth factor (EGF) to changes in tympanic membrane perforation diameter. J Med Sci 2018;18:186-191

14 Lou ZC, Yang J, Tang Y, Fu YH. Topical application of epidermal growth factor with no scaffold material on the healing of human traumatic tympanic membrane perforations. Clin Otolaryngol 2016;41(6):744-749

15 Zhengcai-Lou, Zihan-Lou, Yongmei-Tang. Comparative study on the effects of EGF and bFGF on the healing of human large traumatic perforations of the tympanic membrane. Laryngoscope 2016;126(1):E23-E28
16 Ramsay HA, Heikkonen EJ, Laurila PK. Effect of epidermal growth factor on tympanic membranes with chronic perforations: a clinical trial. Otolaryngol Head Neck Surg 1995;113(4):375-379

17 Jian-Yang, Zi-Han-Lou, Yahui-Fu, Zheng-Cai-Lou. A retrospective study of EGF and ofloxacin drops in the healing of human large traumatic eardrum perforation. Am J Otolaryngol 2016;37(4):294-298 